Pacific Journal of Mathematics

A THEOREM OF LITTLEWOOD AND LACUNARY SERIES 


\title{
A THEOREM OF LITTLEWOOD AND LACUNARY SERIES FOR COMPACT GROUPS
}

\author{
Alessandro Figà-Talamanca and Daniel Rider
}

Let $G$ be a compact group and $f \in L^{2}(G)$. We prove that given $p<\infty$ there exists a unitary transformation $U$ of $L^{2}(G)$ into $L^{2}(G)$, which commutes with left translations and such that $U f \in L^{p}$. The proof is based on techniques developed by $\mathrm{S}$. Helgason for a similar question. The result stated above, which is an extension of a theorem of Littlewood for the unit circle is then applied to the study of lacunary Fourier series.

The following two results concerning Fourier series of functions defined on the unit circle were proved by Littlewood [5]:

I. Suppose that for any choice of complex numbers $\alpha_{n}$, with $\left|\alpha_{n}\right|=1, \sum \alpha_{n} a_{n} e^{i n x}$ is the Fourier series of an integrable function (or a Fourier-Stieltjes series) then $\sum\left|a_{n}\right|^{2}<\infty$.

II. Let $\sum\left|a_{n}\right|^{2}<\infty$. Then given $p<\infty$ there exist complex numbers $\alpha_{n}$, with $\left|\alpha_{n}\right|=1$, such that $\sum \alpha_{n} a_{n} e^{i n x}$ is the Fourier series of a function in $L^{p}$.

Helgason [3] has generalized I to Fourier series on compact groups. Let $G$ be a compact group with normalized Haar measure $d x$. If $f \in L^{1}(G)$ then $f$ is uniquely represented by a Fourier series

$$
f(x) \sim \sum_{\gamma \in \Gamma} d_{\gamma} \operatorname{Tr}\left(A_{\gamma} D_{\gamma}(x)\right)
$$

where $\operatorname{Tr}$ denotes the usual trace, $\Gamma$ is the set of equivalence classes of irreducible unitary representations of $G, D_{\gamma}$ is a representative of the class $\gamma, d_{\gamma}$ is the degree of $\gamma$, and $A_{\gamma}$ is the linear transformation given by

$$
A_{\gamma}=\int_{G} f(x) D_{\gamma}\left(x^{-1}\right) d x .
$$

Helgason has proved

I'. Suppose that, for any choice of unitary transformations $U_{\gamma}$ on the Hilbert space of dimension $d_{\gamma}, \sum_{\gamma \in \Gamma} d_{\gamma} \operatorname{Tr}\left(U_{\gamma} A_{\gamma} D_{\gamma}(x)\right)$ is the Fourier series of an integrable function (or a Fourier-Stieltjes series) then

Received April 1, 1965. This research was supported in part by Air Force Office of Scientific Research Grant A-AFOSR 335-63. 


$$
\sum_{\gamma \in \Gamma} d_{\gamma} \operatorname{Tr}\left(A_{\gamma} A_{\gamma}^{*}\right)<\infty
$$

In view of the Schur-Peter-Weyl formula

$$
\int_{G}|f(x)|^{2} d x=\sum_{\gamma \in \Gamma} d_{\gamma} \operatorname{Tr}\left(A_{\gamma} A_{\gamma}^{*}\right)
$$

Helgason's result is an extension of I.

In this paper, using Helgason's techniques, we propose to extend II to compact groups in the same sense. That is we prove

II'. Let $\sum d_{\gamma} \operatorname{Tr}\left(A_{\gamma} A_{\gamma}^{*}\right)<\infty$. Given $p<\infty$ there exist unitary transformations $U_{\gamma}$ such that $\sum d_{\gamma} \operatorname{Tr}\left(U_{\gamma} A_{\gamma} D_{\gamma}(x)\right)$ is the Fourier series of a function in $L^{p}$.

This is accomplished as in [3] by proving and exploiting the "lacunarity" of a certain subset of the space of irreducible unitary representations of the product group $\Pi_{i \in S} \boldsymbol{U}\left(d_{i}\right)$ where $\boldsymbol{U}\left(d_{i}\right)$ is the group of unitary transformations of the Hilbert space of dimension $d_{i}$ and $S$ is an arbitrary index set. In the last section we discuss in general lacunary properties of subsets of the space of irreducible representations of a compact group.

2. The main result. For a positive integer $n$ let $\boldsymbol{U}(n)$ be the group of unitary transformations of the Hilbert space of dimension $n$. The normalized Haar measure on $\boldsymbol{U}(n)$ will be denote by $d V$.

Lemma 1. Let $A$ be an $n \times n$ matrix. Then for $s=1,2,3, \cdots$

$$
\int_{U(n)}|\operatorname{Tr}(A V)|^{2 s} d V \leqq \frac{B(s)}{n^{s}}\left[\operatorname{Tr}\left(A A^{*}\right)\right]^{s}
$$

where $B(s)$ is a constant depending only on $s$.

Proof. Since $d V$ is left and right invariant it is sufficient to prove the lemma when $A$ is diagonal. Letting $\boldsymbol{e}_{1}, \boldsymbol{e}_{2}, \cdots, \boldsymbol{e}_{n}$ be a basis for the Hilbert space on which $A$ and $V$ act and $a_{i}=\left\langle A \boldsymbol{e}_{i}, \boldsymbol{e}_{i}\right\rangle, v_{i}=\left\langle V \boldsymbol{e}_{i}, \boldsymbol{e}_{i}\right\rangle$ we have

$$
\begin{aligned}
& \int_{\boldsymbol{U}(n)}|\operatorname{Tr}(A V)|^{2 s} d V=\sum a_{i_{1}} \bar{a}_{i_{2}} a_{i_{3}} \bar{a}_{i_{4}} \cdots a_{i_{2 s-1}} \bar{a}_{i_{2 s}} \\
& \cdot \int_{\boldsymbol{U}(n)} v_{i_{1}} \bar{v}_{i_{2}} \cdots v_{i_{2 s-1}} \bar{v}_{i_{2 s}} d V,
\end{aligned}
$$

where the sum extends over all $i_{1}, i_{2}, \cdots, i_{2 s}$ such that $1 \leqq i_{j} \leqq n$. Each integral in the sum is of the form 


$$
\int_{\boldsymbol{U} \backslash n)} v_{1}^{j_{1}} \bar{v}_{1}^{k_{1}} \cdots v_{n}^{j_{n}} \bar{v}_{n}^{k_{n}} d V
$$

Now each such integral is zero unless $j_{1}=k_{1}, \cdots, j_{n}=k_{n}$. For let $W$ be a diagonal unitary matrix with elements $\alpha_{i}$ of modulus one on the main diagonal. Then by the invariance of $d V$, (3) becomes

$$
\begin{aligned}
\int_{\boldsymbol{U}(n)} & \prod_{i=1}^{n}\left\langle V \boldsymbol{e}_{i}, \boldsymbol{e}_{i}\right\rangle^{j_{i}}\left\langle\boldsymbol{e}_{i}, V \boldsymbol{e}_{i}\right\rangle^{k_{i}} d V \\
& =\int_{\boldsymbol{U}(n ;} \prod_{i=1}^{n}\left\langle W V \boldsymbol{e}_{i}, \boldsymbol{e}_{i}\right\rangle^{j_{i}}\left\langle\boldsymbol{e}_{i}, W V \boldsymbol{e}_{i}\right\rangle^{k_{i}} d V \\
& =\prod_{i=1}^{n} \alpha_{i}^{j_{i}-k_{i}} \int_{\boldsymbol{U}^{(n)}} \prod_{i=1}^{n}\left\langle V \boldsymbol{e}_{i}, \boldsymbol{e}_{i}\right\rangle^{j_{i}}\left\langle\boldsymbol{e}_{i}, V \boldsymbol{e}_{i}\right\rangle^{k_{i}} d V .
\end{aligned}
$$

Thus if the integral is not zero, $\prod_{i=1}^{n} \alpha_{i}^{j_{i}-k_{i}}=1$, for all choices of the $\alpha_{i}$. Clearly this is possible only if $j_{1}=k_{1}, \cdots, j_{n}=k_{n}$. Thus the sum (2) is equal to

$$
\left.\sum\left|a_{i_{1}}\right|^{2} a_{i_{2}}\right|^{2} \cdots\left|a_{i_{s}}\right|^{2} \int_{U(n)}\left|v_{i_{1}}\right|^{2} \cdots\left|v_{i_{s}}\right|^{2} d V
$$

We shall see that for each integer $s$

$$
\int_{U^{(n)}}\left|v_{i}\right|^{2 s} d V \leqq \frac{B(s)}{n^{s}} \quad(s=1,2, \cdots)
$$

where $B(s)$ depends only on $s$. It then fóllows from Hölder's inequality that the integrals in (4) are bounded by $B(s) / n^{s}$ so that (4) is majorized by

$$
\frac{B(s)}{n^{s}} \sum\left|a_{i_{1}}\right|^{2} \cdots\left|a_{i_{s}}\right|^{2}=\frac{B(s)}{n^{s}}\left[\operatorname{Tr}\left(A A^{*}\right)\right]^{s},
$$

and the lemma will be proved.

It is sufficient to calculate (5) for $i=1$. Let $U_{1}(n-1)$ be the subgroup $\left\{T \in \boldsymbol{U}(n): T \boldsymbol{e}_{1}=\boldsymbol{e}_{1}\right\}$. The space $\boldsymbol{U}(n) / \boldsymbol{U}_{1}(n-1)$ of left cosets $\left\{\widetilde{V}=V \boldsymbol{U}_{1}(n-1): V \in \boldsymbol{U}(n)\right\}$ can be identified with the unit sphere $\Sigma_{n}$ in a complex $n$-dimensional Hilbert space. Since $v_{1}$ is constant on these cosets

$$
\int_{\boldsymbol{U}^{(n)}}\left|v_{1}\right|^{2 s} d V=\int_{\Sigma_{n}}\left|\left\langle V \boldsymbol{e}_{1}, \boldsymbol{e}_{1}\right\rangle\right|^{2 s} d \tilde{V}=\int_{\left|w_{1}\right|^{2}+\cdots+\left|w_{n}\right|^{2}=1}\left|w_{1}\right|^{2 s} d \tilde{V}
$$

where $d \tilde{V}$ is the unique normalized measure on $\Sigma_{n}$ invariant with respect to $\boldsymbol{U}(n)$ and

$$
V \boldsymbol{e}_{1}=w_{1} \boldsymbol{e}_{1}+\cdots+w_{n} \boldsymbol{e}_{n} .
$$

If we identify $\Sigma_{n}$ with the real $(2 n-1)$ dimensional sphere $S^{2 n-1}$ in real $2 n$-dimensional space and $d \widetilde{V}$ with $d w$, the normalized invariant 
measure on $S^{2 n-1}$, then

$$
\int_{U(n)}\left|v_{1}\right|^{2 s} d V=\int_{x_{1}^{2}+\cdots+x_{2 n}^{2}=1}\left(x_{1}^{2}+x_{2}^{2}\right)^{8} d w
$$

By Minkowski's inequality and the invariance of $d w(6)$ is bounded by

$$
2^{s} \int_{S^{2 n-1}} x_{1}^{28} d w=2^{s} \frac{\Omega\left(S^{2 n-2}\right)}{\Omega\left(S^{2 n-1}\right)} \int_{-1}^{1} x_{1}^{2 s}\left(1-x_{1}^{2}\right)^{n-1}\left(1-x_{1}^{2}\right)^{-1 / 2} d x_{1}
$$

where $Q\left(S^{m}\right)=\frac{2 \pi^{(m+1) / 2}}{\Gamma\left(\frac{m+1}{2}\right)}$ is the Euclidean surface area of the real $m$-dimensional unit sphere. Thus the integral in (6) is bounded by

$$
2^{s} \frac{2 \pi^{(2 n-1) / 2}}{\Gamma\left(n-\frac{1}{2}\right)} \cdot \frac{\Gamma(n)}{2 \pi^{(2 n) / 2}} \cdot \frac{\Gamma\left(s+\frac{1}{2}\right) \Gamma\left(n-\frac{1}{2}\right)}{\Gamma(n+s)} \leqq \frac{B(s)}{n^{s}}
$$

which proves (5).

COROLLARY 2. Let $J$ be the canonical representation $U \rightarrow U$ of $U(n)$ and $J_{s, t}$ be the tensor product of $J, s$ times and $\widetilde{J}$, the conjugate representation, $t$ times. $J_{s, t}$ decomposes into at most $B(s+t)$ irreducible components. If $s \neq t$ then none of the components is the identity representation.

Proof. If $\chi_{r}$ is the character of the representation $T$, then $\chi_{J_{s, t}}(V)=\left(\chi_{J}(V)\right)^{s} \overline{\left(\chi_{J}(V)\right)^{t}}=(\operatorname{Tr}(V))^{s} \overline{(\operatorname{Tr}(V))^{t}}$. Thus by the lemma

$$
\int_{U(n)}\left|\chi_{s_{s, t}}(V)\right|^{2} d V \leqq B(s+t),
$$

which proves the first statement.

The number of times the identity representation occurs in $J_{s, t}$ is

$$
\int_{U(n)} \chi_{J_{s, t}}(V) d V=\int_{U(n)}(\operatorname{Tr}(V))^{s} \overline{(\operatorname{Tr}(V))^{t}} d V=0
$$

if $s \neq t$ by the statement following (3).

LEMMA 3. Let $\boldsymbol{G}=\Pi_{i \in S} \boldsymbol{U}\left(d_{i}\right)$ be a product of unitary groups $\boldsymbol{U}\left(d_{i}\right)$. Let $F(V)$ be a function on $G$ of the form

$$
F(V)=\sum_{i \in S} d_{i} \operatorname{Tr}\left(A_{i} V_{i}\right)
$$

where $A_{i}$ is a $d_{i} \times d_{i}$ matrix and $V_{i}$ is the projection of $V$ on $\boldsymbol{U}\left(d_{i}\right)$. Then 


$$
\int_{G}|F(V)|^{2 s} d V \leqq B(s)\left(\int|F(V)|^{2} d V\right)^{s}
$$

where $d V$ is the normalized Haar measure on $G$.

Proof. It suffices to prove the lemma when

$$
F(V)=\sum_{i=1}^{N} d_{i} \operatorname{Tr}\left(A_{i} V_{i}\right)
$$

Then

$$
\begin{aligned}
& \int_{G}|F(V)|^{2 s} d V \\
& \quad=\sum \int_{U\left(d_{i}\right) \times \cdots \times U\left(d_{n}\right)} d_{i_{1}} \operatorname{Tr}\left(A_{i_{1}} V_{i_{1}}\right) d_{i_{2}} \overline{\operatorname{Tr}\left(A_{i_{2}} V_{i_{2}}\right)} \cdots d_{i_{2 s}} \overline{\left(A_{i_{2 s}} V_{i_{2 s}}\right)} d V
\end{aligned}
$$

where the sum extends over all $i_{1}, \cdots, i_{2 s}$ such that $1 \leqq i_{j} \leqq N$. By the corollary the only terms in the sum which do not vanish are those of the form

$$
\int_{\boldsymbol{U}\left(d_{i}\right) \times \cdots \times U^{\left(d_{n}\right)}} d_{i_{1}}^{2}\left|\operatorname{Tr}\left(A_{i_{1}} V_{i_{1}}\right)\right|^{2} \cdots d_{i_{s}}^{2}\left|\operatorname{Tr}\left(A_{i_{s}} V_{i_{s}}\right)\right|^{2} d V
$$

By Hölder's inequality (8) is majorized by

$$
d_{i_{1}}^{2} \cdots d_{i_{s}}^{2}\left[\int_{U\left(d_{i_{1}}\right)}\left|\operatorname{Tr}\left(A_{i_{1}} V_{i_{1}}\right)\right|^{2 s} d V_{i_{1}}\right]^{1 / s} \cdots\left[\int_{U\left(d_{i_{s}}\right)}\left|T_{r}\left(A_{i_{s}} V_{i_{s}}\right)\right|^{2 s} d V_{i_{s}}\right]^{1 / s} .
$$

which by Lemma 1 is majorized by

$$
d_{i_{1}}^{3} \cdots d_{i_{s}}^{2} B(s) \frac{\operatorname{Tr}\left(A_{i_{1}} A_{i_{1}}^{*}\right)}{d_{i_{1}}} \cdots \frac{\operatorname{Tr}\left(A_{i_{s}} A_{i_{s}}^{*}\right)}{d_{i_{s}}}
$$

Hence the left side of (7) is bounded by

$$
B(s)\left[\sum_{i}^{N} d_{i} \operatorname{Tr}\left(A_{i} A_{i}^{*}\right)\right]^{s}=B(s)\left[\int_{G}|f(V)|^{2} d V\right]^{s},
$$

where the equality follows from the Peter-Weyl formula.

Now let $G$ be an arbitrary compact group and $\Gamma$ be the set of equivalence classes of irreducible representations of $G$. Let $d_{\gamma}$ be the degree of the class $\gamma$. Then $G=\Pi_{\gamma \in \Gamma} U\left(d_{\gamma}\right)$ is a compact group which can be thought of as the group of unitary transformations of $L^{2}(G)$ into $L^{2}(G)$ which commute with left translations. That is, if $V$ is such a transformation then $V$ corresponds to the element $\left\{V_{\gamma}\right\} \in G$ such that

$$
V f(x) \sim \sum_{\gamma \in \Gamma} d_{\gamma} \operatorname{Tr}\left(V_{\gamma} A_{\gamma} D_{\gamma}(x)\right)
$$

whenever $f(x) \sim \sum_{\gamma \in \Gamma} \operatorname{Tr}\left(A_{\gamma} D_{\gamma}(x)\right) \in L^{2}(G)$. 
THEOREM 4. Let $f \in L^{2}(G)$ and $p<\infty$, then for almost every $V \in G, V f \in L^{p}(G)$.

Proof. Let $V f(x)=f(V, x)=\sum d_{\gamma} \operatorname{Tr}\left(V_{\gamma} A_{\gamma} D_{\gamma}(x)\right)$. Then $f(V, x)$ can be considered as a function on $G \times G$. For fixed $x \in G$ we have by Lemma 2 that

$$
\begin{aligned}
\int_{G}|f(V, x)|^{2 s} d V \leqq B(s)\left[\int_{G}|f(V, x)|^{2} d V\right]^{s} & =B(s)\left[\left.\sum_{\gamma \in \Gamma} d_{\gamma} \operatorname{Tr}\left(A_{\gamma} A_{\gamma}^{*}\right)\right|^{s}\right. \\
& =B(s)\left[\int_{G}|f(x)|^{2} d x\right]^{s},
\end{aligned}
$$

so that

$$
\begin{aligned}
\int_{G} \int_{G}|f(V, x)|^{28} d x d V & =\int_{G} \int_{G}|f(V, x)|^{2 s} d V d x \\
& \leqq B(s)\left[\int_{G}|f(x)|^{2} d x\right]^{s} .
\end{aligned}
$$

Therefore if $f \in L^{2}(G)$, then for almost every $V \in G, \int_{G}|V f(x)|^{28} d x<\infty$. Letting $s>p / 2$ we obtain the theorem.

We remark for later use that for some $V$

$$
\|V f\|_{p} \leqq\|V f\|_{2 s} \leqq 2 B(s)\|f\|_{2} \text {. }
$$

Indeed the set of $V$ for which

$$
\int_{G}|V f(x)|^{2 s} d x>2 B(s)\left[\int_{G}|f(x)|^{2} d x\right]^{s}
$$

cannot be of measure one.

We will also use the following

REMARK 5. Let $f \in C(G)$ be a continuous function such that for all self adjoint $V \in G, V f \in C(G)$, then $f(x) \sim \sum d_{\gamma} \operatorname{Tr}\left(A_{\gamma} D_{\gamma}(x)\right)$ with $\sum d_{\gamma} \operatorname{Tr}\left(\left|A_{\gamma}\right|\right)<\infty\left(\left|A_{\gamma}\right|\right.$ is the absolute value of the matrix $\left.A_{\gamma}\right)$. Indeed letting $\widetilde{f}(x)=\widetilde{f\left(x^{-1}\right)}$ we can write $f=(f+\widetilde{f}) / 2+i(f-\widetilde{f}) / 2 i=$ $f_{1}+i f_{2}$. If $f_{i}(x) \sim \sum d_{\gamma} \operatorname{Tr}\left(A_{\gamma},{ }_{i} D_{\gamma}(x)\right) \quad(i=1,2) \quad$ then $A_{\gamma, i}^{*}=A_{\gamma, i}$. Therefore there exists a self adjoint $V=\left\{V_{\gamma}\right\} \in G$ such that $A_{\gamma, i} V_{\gamma}=$ $\left|A_{\gamma, i}\right|$. Thus $\sum d_{\gamma} \operatorname{Tr}\left(\left|A_{\gamma, i}\right| D_{\gamma}(x)\right)$ is continuous so that applying a method of summation as in $[4,8.3]$ we obtain that the partial sums of $\sum d_{\gamma} \operatorname{Tr}\left(\left|A_{\gamma, i}\right|\right)=\sum d_{\gamma} \operatorname{Tr}\left(\left|A_{\gamma, i}\right| D_{\gamma}(e)\right)$ are bounded. Thus $\sum d_{\gamma} \operatorname{Tr}\left(\left|A_{\gamma}\right|\right) \leqq$ $\sum d_{\gamma} \operatorname{Tr}\left(\left|A_{\gamma, 1}\right|\right)+\sum d_{y} \operatorname{Tr}\left(\left|A_{\gamma, 2}\right|\right)<\infty$.

We shall call a series $\sum d_{\gamma} \operatorname{Tr}\left(A_{\gamma} D_{\gamma}(x)\right)$ satisfying $\sum d_{\gamma} \operatorname{Tr}\left(\left|A_{\gamma}\right|\right)<\infty$ an absolutely convergent series. The space of such functions will be denoted by $A(G)$. It is easy to see that $A(G)$ consists of functions of the type $f * g$ with $f, g \in L^{2}(G)$. The space $A(G)=L^{2}(G) * L^{2}(G)$ has been 
studied in [1].

3. Lacunary Fourier series. Given a compact group $G$ we shall say that a subset $E \subseteq \Gamma$ of the set of irreducible unitary representation of $G$ is a Sidon set if it satisfies the following property:

A. $\sum d_{\gamma} \operatorname{Tr}\left(\left|A_{\gamma}\right|\right)<\infty$ whenever $\sum_{\gamma \in E} d_{\gamma} \operatorname{Tr}\left(A_{\gamma} D_{\gamma}(x)\right)$ is the Fourier series of a continuous function (cf. $[6,5.7])$.

$A$ set $E \subset I^{r}$ will be called a set of type $\Lambda(p)$ (or $E \in \Lambda(p)$ ) for $p>1$ if it satisfies

B. If $\sum_{\gamma \in_{E}} d_{\gamma} \operatorname{Tr}\left(A_{\gamma} D_{\gamma}(x)\right)$ is the Fourier series of an integrable function then it is the Fourier series of a function in $L^{p}$ (cf. [8]).

If $B$ is a space of functions on $G$ and $E \cong \Gamma$ we will denote by $B_{E}$ those functions in $B$ with a series of the form $\sum_{\gamma \in E} d_{\gamma} \operatorname{Tr}\left(A_{\gamma} D_{\gamma}(x)\right)$. It is seen as in $[8,1.4]$ that $E \in \Lambda(p)$ if, for some $r<p, L_{E}^{r}=L_{E}^{p}$. Clearly $\Lambda\left(p_{1}\right) \subseteq \Lambda\left(p_{2}\right)$ if $p_{1} \geqq p_{2}$.

If $\boldsymbol{G}=\prod_{i \in S} \boldsymbol{U}\left(d_{i}\right)$ then $S$ can be thought of as the set of irreducible representations of $\boldsymbol{G}$ consisting of the projections of $\boldsymbol{G}$ onto the $\boldsymbol{U}\left(d_{i}\right)$. Lemma 2 shows that $S \in \Lambda(p)$ for every $p<\infty$. It is a simple matter to prove that $S$ is also a Sidon set. Indeed, if $f(V)=\sum_{i \in S} d_{i} \operatorname{Tr}\left(A_{i} V_{i}\right)$ is a continuous function belonging to $C_{S}(G)$ and if $U=\left\{U_{i}\right\} \in G$ then

$$
U f(V)=\sum_{i \in S} d_{i} \operatorname{Tr}\left(A_{i} U_{i} V_{i}\right)=\text { left translation of } f \text { by } U,
$$

is also continuous. It suffices to pick the $U_{i}$ so that $A_{i} U_{i}=\left|A_{i}\right|$ to obtain that $\sum_{i \in S} d_{i} \operatorname{Tr}\left(\left|A_{i}\right|\right)<\infty$.

We shall now establish a characterization of sets of type $\Lambda(p)$ which will imply that every Sidon set is a $A(p)$ set for every $p<\infty$. For a group $G$ denote by $\mathscr{R}_{p}=\mathscr{R}_{p}(G)$ the algebra of operators on $L^{p}(G)$ generated in the weak operator topology by the operators $\left\{R_{y}: y \in G\right\}$ where $R_{y} f(x)=f(x y)$. We shall use the fact [2, Th. 6] that $\mathscr{R}_{p}$ is (isometric and isomorphic to) the dual space of a Banach space $A^{p}$ of continuous functions on $G . A^{2}=A(G)$ the space of functions with absolutely convergent Fourier series [1].

The isomorphism between $\mathscr{R}_{p}$ and the dual space of $A^{p}$ is given by $T \rightarrow \varphi_{T}$ where $\varphi_{T}(f)=T f(e)$. This correspondence is well defined because every $T \in \mathscr{R}_{p}$ maps each element of $A^{p}$ into a continuous function, indeed an element of $A^{p}$. We also have that $\mathscr{P}_{p}$ consists exactly of those bounded operators on $L^{p}$ which commute with left translations.

Now if $T \in \mathscr{R}_{p}, p>2$, then $T \in \mathscr{R}_{2}$ and $\|T\|_{\mathscr{R}_{2}} \leqq M\|T\|_{\mathscr{c}_{p}}$ where $M$ is a constant depending only on $p$. For if $f \in L^{2}$ then by Theorem 
3 there exists a unitary transformation $U$ commuting with right translations (and therefore with elements of $\mathscr{R}_{p}$ ) such that $U f \in L^{p}$. We can also choose $U$ such that $\|U f\|_{p} \leqq 2 B(s)\|f\|_{2}$ where $B(s)$ is the constant appearing in Lemma 1 and $s>p / 2$ (cf. the remarks following the proof of Theorem 4).

We then have that $T U f \in L^{p}$ and $U^{*} T U f=T U^{*} U f=T f \in L^{2}$. Also $\|T f\|_{2}=\left\|U^{*} T U f\right\|_{2} \leqq\|T U f\|_{2} \leqq\|T U f\|_{p} \leqq\|T\|_{\mathscr{R}_{p}}\|U f\|_{p} \leqq\|T\|_{\mathscr{T}_{p}}$ $M\|f\|_{2}$ where $M=2 B(s)$. Therefore $\|T\|_{\Omega_{2}} \leqq M\|T\|_{\Re_{p}}$. This implies that $A^{2} \leqq A^{p}$ and \|\|$_{A^{p}} \leqq M\|\|_{A^{2}}$. It is now a simple matter to prove:

THEOREM 6. Let $E \cong \Gamma$ be a set of irreducible unitary representations of $G$ and $p>2$. The following are equivalent:

(a) $E$ is a set of type $\Lambda(p)$.

(b) If $T \in \mathscr{R}_{2}$ there exists $S \in \mathscr{R}_{p}$ such that $T f=S f$ for all $f \in L_{E}^{p}$. (c) If $f \in A_{E}^{p}$ then $f \in A^{2}=A(G)$.

(d) Every closed subspace of $L_{E}^{p}$ which is invariant under left translations is the range of a projection $P$ belonging to $\mathscr{R}_{p}$ which is self-adjoint in the sense that $P_{\gamma}=P_{\gamma}^{*}$ for each $\gamma \in \Gamma$.

Proof. Let $E \in \Lambda(p)$. Then $L_{E}^{p}=L_{E}^{2}$ so that by the open mapping theorem there exists $B$ such that $\|f\|_{p} \leqq B\|f\|_{2}$ for $f \in L_{E}^{2}$. As $L_{E}^{2}$ is invariant under right and left translations there exists a projection $P_{E}$ of $L^{2}$ onto $L_{E}^{2}$ which commutes with right and left translations. If $T \in \mathscr{R}_{2}$ let $S=T P_{E}$, then $\|S f\|_{p} \leqq\|T\|\left\|P_{E} f\right\|_{p} \leqq$ $\|T\| B\left\|P_{E} f\right\|_{2} \leqq B\|T\|\|f\|_{p}$. Thus $S \in \mathscr{R}_{p}$ and (a) implies (b).

Now assume (b) holds. If $f \in L_{E}^{2}$ then by Theorem 3 there exists $U \in \mathscr{R}_{2}$ such that $U f \in L^{p}$; clearly $U f \in L_{E}^{p}$. Let $S \in \mathscr{R}_{p}$ be such that $S g=U^{*} g$ for all $g \in L_{E}^{p}$; then $S U f=U^{*} U f=f \in L^{p}$. Hence $L_{E}^{p}=L_{E}^{2}$ so that (b) implies (a).

We now show that (a) and (b) imply (d). Indeed if (a) holds the projection $P_{E}$ of $L^{2}$ onto $L_{E}^{2}$ is bounded in $L^{p}$. Suppose the $Y \subseteq L_{E}^{p}$ is invariant under left translations, let $P_{Y}$ be the projection (belonging to $\mathscr{R}_{2}$ ) of $L^{2}$ onto the left invariant subspace of $L^{2}$ generated by $Y$. By (b) there exists $S \in \mathscr{R}_{p}$ with $S=P_{Y}$ on $L_{E}^{p}$. Then $P_{E} S=P_{Y}$ so that $P_{Y} \in \mathscr{R}_{p}$.

Suppose (d) holds and let $U$ be a unitary self adjoint element of $\mathscr{R}_{2}$. Then $U^{2}=I$ so that $P=(U+I) / 2$ is a projection which commutes with left translations. Let $Y$ be the subspace of $L_{E}^{p}$ generated by $P L_{E}^{2} \cap L_{E}^{p}$. Then $Y$ is invariant under left translations so that by (d) there is a self-adjoint projection of $L^{p}$ onto $Y$ commuting with left translations. Clearly this projection is $P P_{E}$ so that $P P_{E} \in \mathscr{R}_{p}$. Hence $U P_{E}=(2 P-I) P_{E} \in \mathscr{R}_{p}$. Therefore $U P_{E} f$ is continuous for every $f \in A^{p}$. In particular if $f(x) \sim \sum_{\gamma \epsilon_{E}} d_{\gamma} \operatorname{Tr}\left(A_{\gamma} D_{\gamma}(x)\right) \in A_{E}^{p}$ then $U P_{E} f=U f$ is 
continuous. Therefore by Remark $4, A_{E}^{p} \subseteq A(G)$.

Finally since $A \subseteq A^{p}$ with $\|f\|_{A^{p}} \leqq M\|f\|_{A}$, (c) implies that $A_{E}=A_{E}^{p}$, so that, by the closed graph theorem, $\|f\|_{A} \leqq B\|f\|_{A^{p}}$ for each $f \in A_{E}^{p}$. Each $T \in \mathscr{R}_{2}$ defines therefore a continuous linear functional on $A_{E}^{p}$ by $T f(e)$. The Hahn-Banach extension of this functional determines, in view of the duality between $A^{p}$ and $\mathscr{R}_{p}$, an element $S \in \mathscr{R}_{p}$ such that $S f(x)=\left(S L_{x} f\right)(e)=\left(T L_{x} f\right)(e)=T f(x)$ for $f \in A_{E}^{p}$; therefore $S=T$ on $L_{E}^{p}$. Thus (c) implies (b) and the theorem is proved.

REMARK 7. It suffices for condition (d) to be true that every closed left invariant subspace of $L_{E}^{p}$ is the range of a projection. Indeed the argument used in [7, Th. 1] will show that such a projection can be chosen to be left invariant (and therefore belonging to $\mathscr{R}_{p}$ ).

THEOREM 8. $E \subseteq \Gamma$ is a Sidon set if and only if for each $T \in \mathscr{R}_{2}$ there exists a finite measure $\mu$ on $G$ such that $T f=f * \mu$ for each $f \in L_{E}^{3}$.

Proof. One applies the same duality argument used in the proof of Theorem 6 (cf. also [6, 5.7.3]. Assume first that $E$ is a Sidon set. Then given $T \in \mathscr{R}_{2}$, define a linear functional $F$ on $C_{E}$ by $F(f)=$ $T f(e)$. Then $F$ is well defined, since $f \in C_{E} \Rightarrow f \in A$; by the closed graph theorem $F$ is continuous and has a Hahn-Banach extension to all of. $C(G)$. That is, by the Riesz representation theorem there exists a bounded measure $\mu$ satisfying

$$
F(f)=\int_{\theta} f\left(x^{-1}\right) d \mu(x) \text { for all } f \in C_{E} .
$$

Since $T$ commutes with left translations $T f=f * \mu$ for all $f \in C_{E}$. Conversely let $E$ satisfy the hypothesis of the theorem, to prove that $E$ is a Sidon set, let $f \in C_{E}$ and let $T$ be an unitary element of $\mathscr{F}_{2}$. By hypothesis there exists a measure $\mu$ such that $T f=f * \mu$. Hence $T f \in C(G)$ and by Remark $5, f \in A$.

\section{Corollary 9. Every Sidon set is a $\Lambda(p)$ set for every $p$.}

Proof. If $\mu$ is a bounded measure and $R_{\mu} f=f * \mu$, then $R_{\mu} \in \mathscr{R}_{p}$ for every $p$. Therefore, by Theorem 8 if $E$ is a Sidon set condition (b) of Theorem 6 holds.

REMARK 10. In [4, 9.2] a sufficient condition for a set $E \subseteq \Gamma$ to be a Sidon set is given. This condition includes the requirements that the degrees of the representations of $E$ be bounded. The fact that for $\Pi_{i \in S} \boldsymbol{U}\left(d_{i}\right), S$ is a Sidon set shows that this requirement is not necessary. 


\section{REFERENCES}

1. P. Eymard, L'algèbre de Fourier d'un group localement compact, Bull. Soc. Math. France 92 (1964), 181-236.

2. A. Figà-Talamanca, Translation invariant operators in $L^{p}$, Duke Math. J. 32 (1965), 495-501.

3. S. Helgason, Topologies of group algebras and a theorem of Littlewood, Trans. Amer. Math. Soc. 86 (1957), 269-283.

4. E. Hewitt and H. S. Zuckerman, Some theorems on lacunary Fourier series with extensions to compact groups, Trans. Amer. Math. Soc. 93 (1939), 1-19.

5. J. E. Littlewood, On the mean value of power series, Proc. London Math. Soc. 25 (1924), 528-556.

6. W. Rudin, Fourier Analysis on Groups, Interscience, New York, 1962.

7. - Projection on invariant subspaces, Proc. Amer. Math. Soc. 13 (1962), 429-432.

8. - Trigonometric series with gaps, J. Math. Mech. 9 (1960), 203-227.

MASSACHUSETTS INSTITUTE OF TEChNOLOGY 


\section{PACIFIC JOURNAL OF MATHEMATICS}

\section{EDITORS}

\author{
H. SAMmLSON \\ Stanford University \\ Stanford, California \\ R. M. BLUMENTHAL \\ University of Washington \\ Seattle, Washington 98105
}

\author{
*J. DUGUNDJI \\ University of Southern California \\ Los Angeles, California 90007
}

RICHARD ARENS

University of California

Los Angeles, California 90024

\section{E. F. BECKENBACH \\ B. H. NEUMANN \\ ASSOCIATE EDITORS}

\section{SUPPORTING INSTITUTIONS}

\author{
UNIVERSITY OF BRITISH COLUMBIA \\ CALIFORNIA INSTITUTE OF TECHNOLOGY \\ UNIVERSITY OF CALIFORNIA \\ MONTANA STATE UNIVERSITY \\ UNIVERSITY OF NEVADA \\ NEW MEXICO STATE UNIVERSITY \\ OREGON STATE UNIVERSITY \\ UNIVERSITY OF OREGON \\ OSAKA UNIVERSITY \\ UNIVERSITY OF SOUTHERN CALIFORNIA
}

\author{
STANFORD UNIVERSITY \\ UNIVERSITY OF TOKYO \\ UNIVERSITY OF UTAH \\ WASHINGTON STATE UNIVERSITY \\ UNIVERSITY OF WASHINGTON \\ * * * \\ AMERICAN MATHEMATICAL SOCIETY \\ CHEVRON RESEARCH CORPORATION \\ TRW SYSTEMS \\ NAVAL ORDNANCE TEST STATION
}

\footnotetext{
Mathematical papers intended for publication in the Pacific Journal of Mathematics should be typewritten (double spaced). The first paragraph or two must be capable of being used separately as a synopsis of the entire paper. It should not contain references to the bibliography. Manu. scripts may be sent to any one of the four editors. All other communications to the editors should be addressed to the managing editor, Richard Arens at the University of California, Los Angeles, California 90024.

50 reprints per author of each article are furnished free of charge; additional copies may be obtained at cost in multiples of 50 .
}

The Pacific Journal of Mathematics is published monthly. Effective with Volume 16 the price per volume (3 numbers) is $\$ 8.00$; single issues, $\$ 3.00$. Special price for current issues to individual faculty members of supporting institutions and to individual members of the American Mathematical Society: $\$ 4.00$ per volume; single issues $\$ 1.50$. Back numbers are available.

Subscriptions, orders for back numbers, and changes of address should be sent to Pacific Journal of Mathematics, 103 Highland Boulevard, Berkeley 8, California.

Printed at Kokusai Bunken Insatsusha (International Academic Printing Co., Ltd.), No. 6, 2-chome, Fujimi-cho, Chiyoda-ku, Tokyo, Japan.

PUBLISHED BY PACIFIC JOURNAL OF MATHEMATICS, A NON-PROFIT CORPORATION

The Supporting Institutions listed above contribute to the cost of publication of this Journal, but they are not owners or publishers and have no responsibility for its content or policies.

* Paul A. White, Acting Editor until J. Dugundji returns. 


\section{Pacific Journal of Mathematics}

\section{Vol. 16, No. $3 \quad$ BadMonth, 1966}

Gert Einar Torsten Almkvist, Stability of linear differential equations with

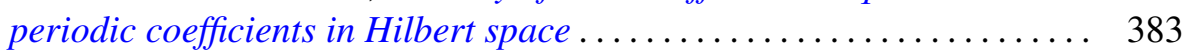

Richard Allen Askey and Stephen Wainger, A transplantation theorem for ultraspherical coefficients ................................ 393

Joseph Barback, Two notes on regressive isols .................. 407

Allen Richard Bernstein and Abraham Robinson, Solution of an invariant subspace problem of K. T. Smith and P. R. Halmos .............. 421

P. R. Halmos, Invariant subspaces of polynomially compact operators . . . . 433

Leon Bernstein, New infinite classes of periodic Jacobi-Perron algorithms.................................... 439

Richard Anthony Brualdi, Permanent of the direct product of matrices .... . 471

W. Wistar (William) Comfort and Kenneth Allen Ross, Pseudocompactness and uniform continuity in topological groups .................. 483

James Michael Gardner Fell, Algebras and fiber bundles . . . . . . . . . . . . 497

Alessandro Figà-Talamanca and Daniel Rider, A theorem of Littlewood and lacunary series for compact groups ..................... 505

David London, Two inequalities in nonnegative symmetric matrices...... 515

Norman Jay Pullman, Infinite products of substochastic matrices ........ 537

James McLean Sloss, Reflection and approximation by interpolation along

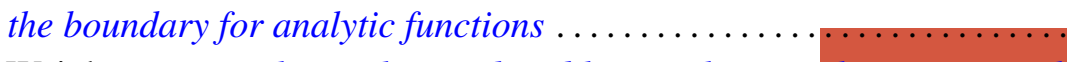

Carl Weinbaum, Visualizing the word problem, with an application to sixth groups................................... 